Kompass

Neumología

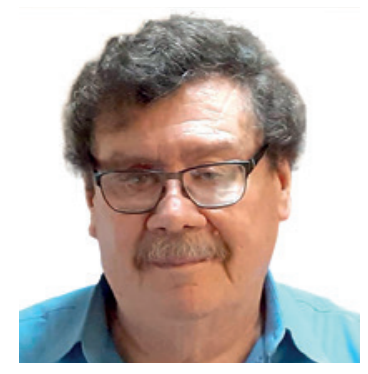

\title{
EPOC: Enfoque personalizado para el tratamiento según la guía GOLD 2021
}

Moisés Acuña-Kaldman

Hospital General del Estado de Sonora, Hermosillo, Sonora, México

Resumen de Muro S, Sugiura H, Darken P, et al.: Efficacy of budesonide/glycopyrronium/formoterol metered dose inhaler in patients with COPD: post-hoc analysis from the KRONOS study excluding patients with airway reversibility and high eosinophil counts. Respir Res. 2021;22(1):187.

\section{Keywords}

COPD · Asthma-like features - Triple therapy · Budesonide . Glycopyrrolate · Formoterol fumarate · KRONOS · Pulmonary function · Exacerbation

\footnotetext{
Abstract

Background: In the Phase III KRONOS study, triple therapy with budesonide/glycopyrronium/formoterol fumarate metered dose inhaler (BGF MDI) was shown to reduce exacerbations and improve lung function versus glycopyrronium/formoterol fumarate dihydrate (GFF) MDI in patients with moderate-to-very severe chronic obstructive pulmonary disease (COPD). However, whether the benefits related to the ICS component of BGF are driven by patients with high blood eosinophil counts (EOS) and/or airway reversibility has not been previously studied.

Methods: KRONOS was a Phase III, double-blind, parallel-group, multicenter, randomized, controlled study of patients with moderate-to-very-severe COPD. Patients were randomized 2:2:1:1 to receive BGF 320/14.4/10 $\mu \mathrm{g}$, GFF 14.4/10 $\mu \mathrm{g}$, budesonide/formoterol fumarate dihydrate (BFF) MDI $320 / 10 \mu \mathrm{g}$ via a single Aerosphere inhaler, or open-label budesonide/formoterol fumarate dihydrate dry powder inhaler 400/12 $\mu \mathrm{g}$ (BUD/FORM DPI; Symbicort Turbuhaler) twice-daily for 24 weeks. Efficacy outcomes included in this post-hoc analysis were change from baseline in morning pre-dose trough FEV1 over weeks 12-24 and the rate of moderate-to-severe and severe COPD exacerbations. Adverse events in the non-reversible subgroup are also reported.
}

Results: Of 1896 patients analyzed, 948 (50\%) were non-reversible and had EOS $<300$ cells $/ \mathrm{mm}^{3}$. In this group, BGF significantly improved morning pre-dose trough $\mathrm{FEV}_{1}$ versus BFF and BUD/ FORM (least squares mean treatment difference, 95\% confidence interval [Cl] $69 \mathrm{~mL}[39,99]$, unadjusted $p<0.0001$ and $51 \mathrm{~mL}[20$, 81], unadjusted $p=0.0011$, respectively) and was comparable to GFF. BGF also significantly reduced annual moderate-to-severe exacerbation rates versus GFF (rate ratio [95\% Cl] 0.53 [0.37, 0.76], unadjusted $p=0.0005$ ), with numerical reductions observed versus BFF and BUD/FORM. These results were similar for the overall study population. Safety findings were generally similar between non-reversible patients with EOS $<300$ cells $/ \mathrm{mm}^{3}$ and the overall population.

Conclusions: In patients with moderate-to-very-severe COPD without airway reversibility and EOS $<300$ cells $/ \mathrm{mm}^{3}$, BGF significantly improved morning pre-dose trough FEV1 versus BFF and BUD/FORM and significantly reduced the rate of moderate-tosevere exacerbations versus GFF. These findings demonstrate that BGF can provide benefits for a broad range of patients with COPD, and that the overall findings of the KRONOS primary analysis were not driven by patients with reversible airflow obstruction or high eosinophil counts.

Trial registration: ClinicalTrials.gov, NCT02497001. Registered 14 July 2015, https://clinicaltrials.gov/ct2/show/NCT02497001

(C) 2021 Muro, Sugiura, Darken, Dorinsky 


\section{Transferencia en la práctica}

\section{Contexto del estudio}

La iniciativa GOLD (Global Initiative for Chronic Obstructive Lung Disease) en sus recomendaciones 2021 ha modificado el tratamiento de pacientes con una enfermedad pulmonar obstructiva crónica (EPOC) con la utilización de broncodilatador en grupo A, beta 2 agonistas de acción prolongada (long-acting beta 2-agonists, $\mathrm{LABA}$ )/antagonistas de acción prolongada (long-acting muscarinic antagonists, LAMA) en grupo B y un LAMA en grupo C, o una combinación LABA+LAMA, en pacientes sintomáticos con un COPD assessment test superior a 20 puntos y un LABA+CSI (corticoesteroides inhalados) en casos de pacientes con eosinófilos (EOS en sangre) superior a 300 en el grupo D. Sin embargo, la Sociedad Respiratoria Japonesa (JRS) solo recomienda tratamientos que contienen CSI para el tratamiento de pacientes con EPOC con características adicionales asociadas con asma. El estudio KRONOS (NCT02497001) es un ensayo aleatorizado, doble ciego y de grupo paralelo de 24 semanas, de fase III, que investigó la eficacia y la seguridad de la combinación triple de budesonida/glicopirronio/formoterol fumarato dihidratado dosis inhalador (BGF MDI) versus las terapias duales correspondientes glicopirronnio/formoterol fumarato dihidratado (GFF) MDI y budesonida/formoterol fumarato dihidratado (BFF) MDI, y budesonida/formoterol fumarato dihidratado de polvo seco inhalador (BUD/FORM DPI) en pacientes de Canadá, China, Japón y los EE. UU.

\section{Resultados del estudio}

Se demostró que el tratamiento con BGF proporciona beneficios sobre la función pulmonar, los síntomas y las exacerbaciones frente a las terapias duales, y se toleró bien en pacientes con EPOC de moderada a muy grave. La eficacia de la terapia triple se ha divulgado previamente en las poblaciones de EPOC que incluyeron a pacientes con algunas características del asma. Se ha observado que la mejoría en las tasas de exacerbación después del tratamiento con CSI en EPOC ocurren en una amplia gama de niveles de EOS en sangre, pero el aumento de la magnitud del efecto fue en relación a el aumento de eosinófilos.

\section{Conclusiones y recomendaciones para la práctica}

Si bien el estudio KRONOS no incluyo a pacientes con un diagnóstico actual de asma, no se excluyeron aquellos con antecedentes de asma, y algunos pacientes tenían ciertas características de la enfermedad que, si bien están presentes en muchos pacientes con EPOC, también pueden ser características clínicas del asma (reversibilidad de las vías respiratorias y/o EOS elevada). Por lo tanto, este análisis post-hoc del estudio KRONOS tuvo como objetivo evaluar la función pulmonar y las exacerbaciones en pacientes con EPOC moderada a muy grave que no tenían reversibilidad en las vías respiratorias y que demostraron Eosinofilia.

En resumen, la estrategia GOLD 2021 abandona el fenotipo mixto (asthma-COPD overlap, ACO) considerando que el asma y EPOC son trastornos diferentes, aunque pueden coexistir en un paciente individual. Si se sospecha un diagnóstico simultáneo de asma, la farmacoterapia debe seguir principalmente las pautas del asma, pero también pueden ser necesarios enfoques farmacológicos y no farmacológicos para la asociación con EPOC. ¿Qué significa esto para el médico tratante? En este estudio, se explora las implicaciones de esta nueva guía sobre el tratamiento de pacientes con enfermedad pulmonar obstructiva crónica, abogando por un enfoque personalizado para el tratamiento, siendo fundamental determinar la reversibilidad, eosinofilia y calidad de vida del paciente en la decisión terapéutica y clasificación de grupo en acuerdo con las guías GOLD 2021.

\section{Disclosure Statement}

Por la referencia el autor declara que no tiene conflicto de interés con respecto a esta transferencia de conocimientos.

\section{Referencias}

1 Global Initiative for Chronic Obstructive Lung Disease: Global strategy for the diagnosis, management, and prevention of chronic obstructive pulmonary disease report 2021. Disponible en https://goldcopd.org/wp-content/ uploads/2019/11/GOLD-2021-REPORT-ver1.0wms.pdf. (Último acceso 2 de Marzo 2021)

2 Yanagisawa S, Ichinose M: Defnition and diagnosis of asthma-COPD overlap (ACO). Allergol Int. 2018;67(2):172-178.

3 Sin DD, Miravitlles M, Mannino DM, et al.: What is asthma-COPD overlap syndrome? Towards a consensus definition from a round table discussion. Eur Respir J. 2016;48(3):664-673.

4 Ferguson GT, Rabe KF, Martinez FJ, et al.: Triple therapy with budesonide/ glycopyrrolate/formoterol fumarate with co-suspension delivery technology versus dual therapies in chronic obstructive pulmonary disease (KRONOS): a double-blind, parallel-group, multicentre, phase 3 randomised controlled trial. Lancet Respir Med. 2018;6(10):747-758.

5 Suissa S, Ariel A: Triple therapy trials in COPD: a precision medicine opportunity. Eur Respir J. 2018;52(6):1801848.

6 Vanfleteren L, Fabbri LM, Papi A, et al.: Triple therapy (ICS/LABA/LAMA) in COPD: time for a reappraisal. Int J Chron Obstruct Pulmon Dis. 2018;13:39713981.

7 Rabe KF, Martinez FJ, Ferguson GT, Wang C, Singh D, Wedzicha JA, et al.: Exacerbation benefit by blood eosinophil counts with budesonide/glycopyrronium/formoterol metered dose inhaler (BGF MDI) at two ICS dose levels in the ETHOS trial: a subgroup analysis. Eur Respir J. 2020;56(suppl. 64):984.

\section{Correspondencia:}

Dr. Moisés Acuña-Kaldman, moisesacunak@gmail.com 